\title{
Attenuation of Microwave Electromagnetic Radiation by Means of Buckypaper
}

\author{
F. F. Komarov ${ }^{a}$, O. V. Mil'chanin ${ }^{a}$, E. Munoz ${ }^{b}$, V. N. Rodionova ${ }^{c}$, \\ V. B. Karpovich ${ }^{a}$, and R. M. Krivosheev ${ }^{a}$ \\ ${ }^{a}$ Sevchenko Research Institute of Applied Physical Problems, Belarusian State University, \\ ul. Kurchatova 7, Minsk, 220108 Belarus \\ ${ }^{b}$ Instituto de Carboquimica (CSIC), Zaragosa, 50018 Spain \\ ${ }^{c}$ Institute for Nuclear Problems, Belarusian State University, Bobruiskaya ul. 11, Minsk, 220030 Belarus \\ *e-mail: KomarovF@bsu.by \\ Received December 1, 2010; in final form, April 5, 2011
}

\begin{abstract}
A material based on carbon nanotubes (buckypaper) is prepared, and the size and structural characteristics of initial nanotubes and the prepared buckypaper are studied. The parameters of electromagnetic radiation-buckypaper interaction are derived for the range 25.5-73.3 GHz. Strong reflection and attenuation of the radiation in this range are discovered.
\end{abstract}

DOI: $10.1134 / \mathrm{S} 1063784211110144$

\section{INTRODUCTION}

Carbon nanotubes (CNTs), nanometer-sized objects, offer a number of unique properties. They are electrically and thermally conductive, strong, and resistive against mechanical and thermal actions $[1,2]$. However, individual carbon nanotubes are hard to handle because of their small size and strong interaction with electric and magnetic fields. This difficulty can be obviated by creating CNT-based macroscopic materials. For example, CNTs are used to make a "paper" in which cellulose fibers are replaced by carbon nanotubes. Such a material is called the buckypaper [3-5]. Here, CNTs combine into bundles owing to van der Waals intermolecular attraction forces and form a stable self-sustained structure due to entwinement of CNT bundles and individual CNTs. Buckypaper retains some properties of CNTs: it is sufficiently strong and conducts high-density electrical current. At the same time, it can be handled as a normal film.

In buckypaper, entwined CNT bunches are distributed randomly as a rule. However, it is possible to provide a pronounced texture in component packing by applying special methods, for example, by depositing a CNT layer in a high magnetic field [4, 6]. Recently, buckypaper was made of an array of oriented CNTs grown on a templet and then packed using the simple and effective method of domino pushing [7]. The properties of buckypaper depend on several factors: CNT growth method and conditions, medium in which CNTs are dissolved, way and time of CNT dispersion in this medium, CNT concentration in the solvent, pressure produced by a vacuum pump when depositing films, and topology and condition of a surface on which the film is deposited [5].
The final mechanical, electrical, and optical performance of buckypaper is influenced by all the above factors and can be modified by subsequent treatments, such as heat treatment in various media and irradiation by high-energy photons [8], electrons [9, 10], or ions [11-14].

The conductivity of buckypaper is limited by weakly bound contacts between CNTs and carrier hopping between individual tubes. Irradiation by highenergy photons or particles causes transitions with covalent bonds between CNTs, which considerably improves the electrical and thermal conductivities of the film (buckypaper) [5]. Note however that both the mechanical and electrical parameters of buckypaper still differ considerably from the unique parameters of individual CNTs or CNT bundles. Young's modulus and rupture strength of buckypaper equal 1-8 GPa and 6-33 MPa, respectively [5]. The magnetic-fieldinduced ordering mentioned above and the use of composites based on polymers with CNTs make it possible to raise Young's modulus to 24-60 GPa [3]. Yet, even these improved values of Young's modulus are less than $10 \%$ of Young's modulus typical of nanotubes.

The unique properties of CNTs and buckypaper make them promising materials for power electronics [15], high thermal conductivity materials, field emission cathodes, efficient cathodes for batteries and capacitors, rf filters [5], sensors used in environmental protection [16], mechanical stress sensors [17], and actuators [18].

Buckypaper and CNT-containing composites are of special interest for designing cheap efficient systems for protection from microwave electromagnetic (EM) 


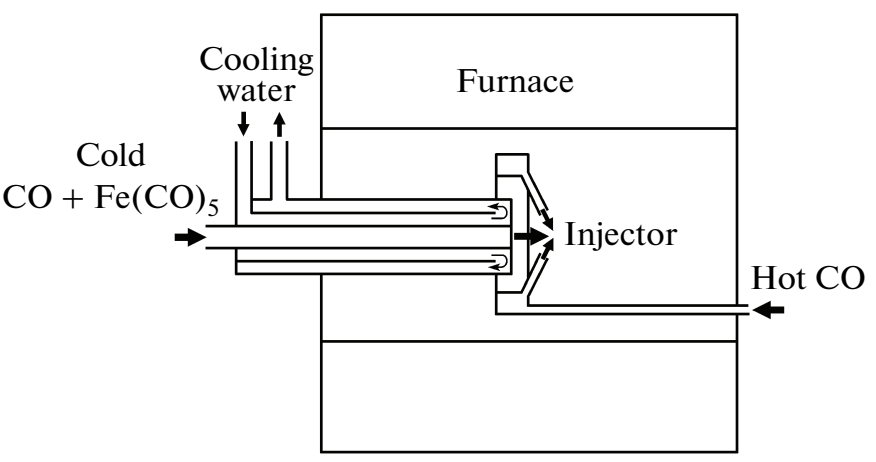

Fig. 1. Schematic of the setup for HiPCO synthesis of single-walled CNTs.

radiation. By this radiation, we mean first of all background EM noise due to cellular phones, microwave ovens, computers, etc. For commercial applications, materials with a screening efficiency of more than $30 \mathrm{~dB}$ (owing to reflection, absorption, and interference quenching) suffice. For military purposes, materials with a much higher EM protection efficiency (from 80 to $100 \mathrm{~dB}$ ) are needed [19].

Different composites (primarily polymer-based composites containing CNTs in an amount of $1-10 \mathrm{wt} \%$ ) have been recently tested as an efficient protecting material $[2,20-30]$. Since buckypaper contains a number of solvent molecules and molecules of a CNT growth catalyst, it can also be viewed as a CNT-based composite. This material attracts attention as a coating protecting unique electronic systems [20], including space-borne electronics. It is of interest in this respect to study the absorption and reflection of EM radiation by buckypaper in a wide wavelength range $[21,31]$. Such information would also be useful for interpretation of data on EM radiation absorption and reflection by multicomponent C NT-containing composites. With this in mind, we prepared buckypaper from single-walled CNTs and studied the absorption and reflection of microwave EM radiation by this material.

\section{PREPARATION AND CHARACTERIZATION OF BUCKYPAPER}

Carbon nanotubes were grown by the $\mathrm{HiPCO}$ (High-Pressure catalytic decomposition of Carbon monOxide) method $[32,33]$, which is a variation of the CVD technique for CNT synthesis. The schematic of the setup for synthesis is depicted in Fig. 1. Iron nanoparticles resulting from the thermal decomposition of iron carbonyl $\mathrm{Fe}(\mathrm{CO})_{5}$ were used as catalysts. In this method, nanotubes form when a $\mathrm{CO}$ flow with a small addition of carbonyl passes through a heated reactor. The thermal decomposition of $\mathrm{Fe}(\mathrm{CO})_{5}$ results in iron clusters acting as catalysts during the Boudouard reaction, which leads to the formation of CNTs,

$$
\mathrm{CO}+\mathrm{CO} \longrightarrow \mathrm{C}_{\mathrm{s}}+\mathrm{CO}_{2} .
$$

Single-walled CNTs were synthesized in a thickwalled quartz tube $2.54 \mathrm{~cm}$ in diameter, which was placed in a furnace (Fig. 1). Part of the tube inside the furnace was kept at $1100^{\circ} \mathrm{C}$, while the inlet and outlet of the tube were maintained at room temperature. A CO- $\mathrm{Fe}(\mathrm{CO})_{5}$ gas mixture was delivered to the tube through a cooled injector. In this way, the gases, on the one hand, were kept at low temperature and, on the other hand, could be rapidly heated when injected to the furnace. The outlet of the injector was surrounded by a ring made up of narrow needles aimed at increasing the heating rate of the gas mixture when hot $\mathrm{CO}$ gas passes through it with a high velocity. To this end, a CO flow was passed through a helical heat exchanger in the furnace. The pressure and flow rate of $\mathrm{CO}$ were $0.2 \mathrm{MPa}$ and $1-2 \mathrm{l} / \mathrm{min}$, respectively. The $\mathrm{Fe}(\mathrm{CO})_{5}$ pressure was equal to $2.5 \mathrm{~Pa}$. Part of the $\mathrm{CO}$ flow was passed through a bubbler filled with $\mathrm{Fe}(\mathrm{CO})_{5}$ and kept approximately at $0^{\circ} \mathrm{C}$. Under such conditions, the partial pressure of $\mathrm{Fe}(\mathrm{CO})_{5}$ was equal to its vapor pressure, $\approx 7 \times 10^{2} \mathrm{~Pa}$. Then, the pressure was decreased to a desired value when mixing with the main $\mathrm{CO}$ flow before entering into the injector. Black disperse reaction products, a mixture of CNTs and carbon-covered iron particles, were deposited on the walls of the tube and outside the furnace.

The synthesized CNTs were examined under a Hitachi-H800 (Japan) transmission electron microscope (TEM) with an operating acceleration voltage of up to $200 \mathrm{kV}$ to determine their size and structural characteristics. Examination was carried out both in the bright-field mode and in the mode of multiplebeam imaging (high-resolution transmission electron microscopy). CNTs in a small amount were placed on copper meshes with an adhesive, dried, and introduced into the column of the microscope.

Raman scattering spectroscopy is an effective and very informative technique for investigating the structure and conductivity type (metallic or semiconductor) of CNTs.

Samples for recording Raman spectra were prepared as follows. Nanotubes and $\mathrm{KBr}$ taken in proportion $1: 20$ (10 $\mathrm{mg}$ of nanotubes and $200 \mathrm{mg} \mathrm{KBr}$ ) was mixed in an agate mortar and compacted under a pressure of $25 \mathrm{MPa}$ for $10 \mathrm{~min}$ to form $2 \mathrm{~mm}$ thick pellets $12 \mathrm{~mm}$ in diameter.

Measurements were taken with an automated spectrometric complex [32] intended for recording Raman spectra. The complex based on a SPEX RAMALOG4 commercial instrument was designed at Sevchenko Research Institute of Applied Physical Problems. Measurements were made in the spectral interval 40$1800 \mathrm{~cm}^{-1}$, and a Raman spectrum was excited by 514.5-nm radiation from an argon laser.

Buckypaper was prepared by ultrasonically dispersing single-walled CNTs in a water solution of Triton X-100 surfactant with subsequent vacuum filtering [33, 34]. In 


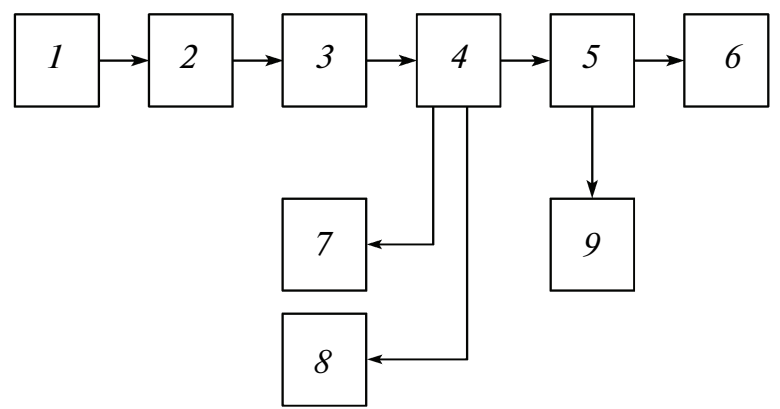

Fig. 2. Scheme for measuring the parameters of nanomaterials in the microwave range: (1) generator, (2) valve, (3) polarization attenuator, (4) directional coupler, (5) measuring line, (6) resonator, (7) digital frequency meter, $(8)$ power meter, and $(9)$ detector unit.

vacuum, dispersed CNTs were passed through a Millipore LS polytetrafluoroethylene membrane filter $5 \mu$ in diameter. As a result, thin ( 25 to $30 \mu \mathrm{m}$ thick) sheets of buckypaper were deposited on the filter. Then, the sheets were dried, removed from the filter, and carefully rinsed in water and methanol taken in large amounts to remove the surfactants. Next, the buckypaper was dried again. The dried buckypaper was annealed at $1000^{\circ} \mathrm{C}$ for $9 \mathrm{~h}$ in an $\mathrm{Ar}$ flow to remove residual solvent and surfactants.

The surface structure and topology of the buckypaper were examined with a Hitachi S-806 scanning electron microscope.

To study interaction between electromagnetic radiation and buckypaper, the buckypaper sheet was placed between the flanges of a waveguide inside which a standing wave was excited. First, measurements were taken without the sheet; then, after the sheet was placed in the waveguide, a mismatch between the electromagnetic radiation and the waveguide was measured. The measurement scheme is schematically depicted in Fig. 2. As parameters characterizing the radiation-material interaction, the electromagnetic radiation attenuation coefficient and the standing wave ratio were used.

Measurements were made in the microwave ranges 25.5-37.8, 36.3-55.7, and 53.7-73.7 GHz. From the relationship $R=(\mathrm{SWR}-1) /(\mathrm{SWR}+1)$, reflection coefficient $R$ of electromagnetic radiation was calculated.

\section{RESULTS AND DISCUSSION}

TEM micrographs of HiPCO-deposited CNTs are shown in Fig. 3. It is seen that individual CNTs combine into bundles, so that the material consists of bundles comprising several tens of nanotubes. Since the CNT material is twisted, it is impossible to exactly measure the length of the bundles. According to estimates, it varies from several hundreds of nanometers to several micrometers. In addition, inclusions, car-

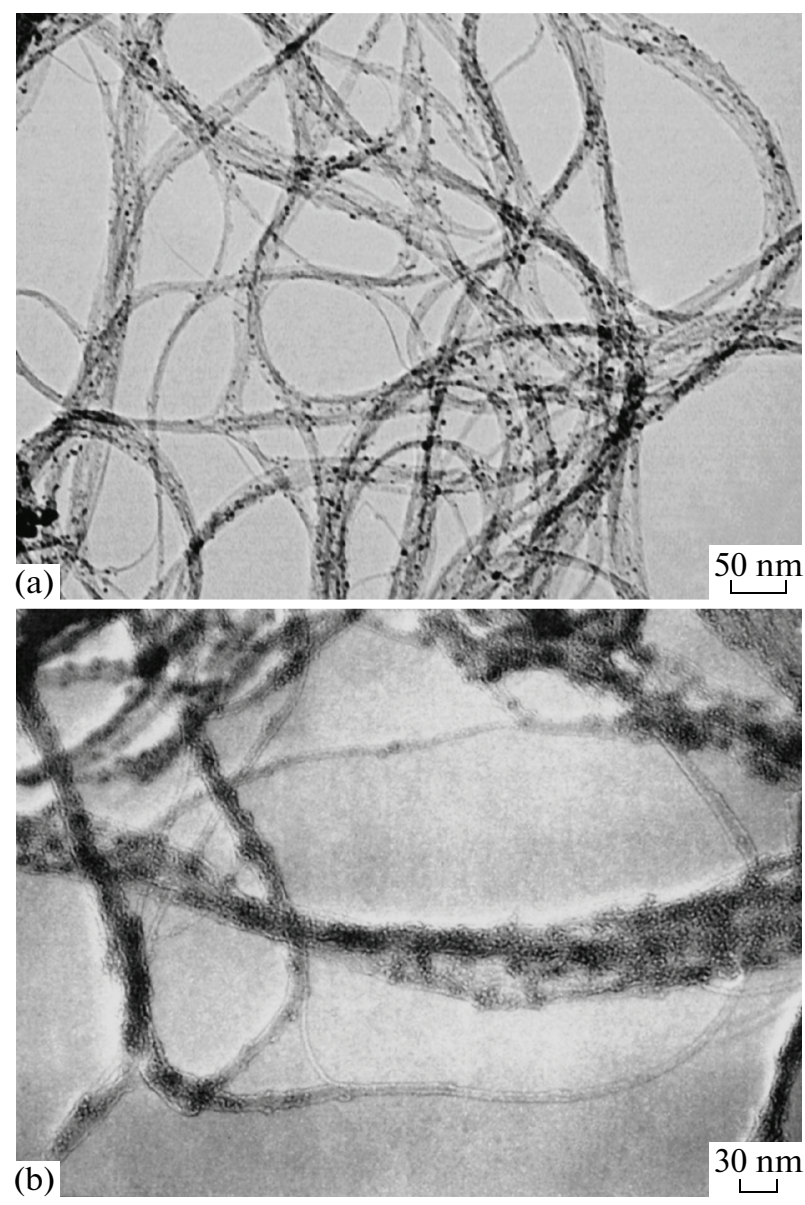

Fig. 3. TEM micrographs of carbon nanotubes obtained by the HiPCO method: (a) bright-field mode and (b) dualbeam imaging mode.

bon nanoclusters and fullerenes, are seen on the surface of the material (a certain amount of inclusions is also observed inside the tubes). The exact statistical analysis of the diameters of individual nanotubes was impossible because of the presence of parallel nanotubes.

It was found by Raman spectroscopy [35-38] that the wavenumbers of radial breathing modes corresponding to intense lines in the range of small wavenumbers $\left(100-300 \mathrm{~cm}^{-1}\right)$ are quite sensitive to the diameter of single-walled CNTs. Hence, studying these modes, one can conveniently determine the CNT diameter distribution in a given array of CNTs. There are models (see [32]) that make it possible to derive an analytical dependence of the radial breathing mode wavenumber on the CNT diameter. Let us use a relationship obtained with regard to van der Waals interaction between CNTs in a bundle [37]: $v_{\mathrm{RBM}}\left(\mathrm{cm}^{-1}\right)=$ $6.5+232 / d(\mathrm{~nm})$, where $v_{\mathrm{RBM}}$ is the radial breathing mode wavenumber.

For the lines at 249 and $267 \mathrm{~cm}^{-1}$ (Fig. 4), the CNT diameters were found to be 0.90 and $0.84 \mathrm{~nm}$, respectively. Thus, we are dealing with single-walled CNTs 


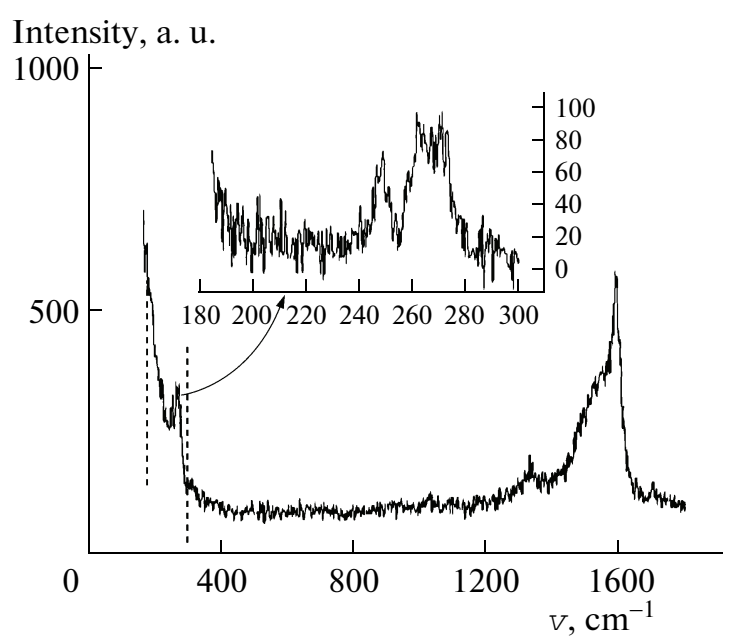

Fig. 4. Spectrum of Raman scattering by carbon nanotubes obtained by vapor-phase CO decomposition.

the diameters of which vary between 0.8 and $0.9 \mathrm{~nm}$. Raman spectral lines in the range of large wavenumbers $\left(1500-1600 \mathrm{~cm}^{-1}\right)$ are assigned to tangential C$\mathrm{C}$ modes. The FWHM of the Raman lines in this range points to the CNT conductivity type $[37,38]$. Narrow lines correspond to resonance in tubes with the semiconductor conductivity, while broad lines correspond to resonance in metallic tubes [35, 39]. In our samples, a wide band of tangential modes with a maximum at $1538 \mathrm{~cm}^{-1}$ was detected. This band correlates with lines in the interval $240-280 \mathrm{~cm}^{-1}$; hence, these lines are assigned to metallic tubes with a small diameter $(\approx 0.9 \mathrm{~nm})$. One more narrow peak at $1593 \mathrm{~cm}^{-1}$ against the background of the wide band is associated with semiconductor tubes. Thus, the samples contain primarily metallic single-walled CNTs and a minor amount CNTs with semiconductor conductivity.

The surface structure of the buckypaper was examined by scanning electron microscopy (SEM). Two images of the surface are shown in Fig. 5. The difference between them is explained by the buckypaper preparation procedure. When the CNT material was deposited on the filters, the lower surface of the buckypaper was smoother and more regular. Spherical inclusions several tens of nanometers in diameter are observed on the surface. These are carbon clusters and iron nanoclusters. It is also seen that CNT bundles intertwine, making the buckypaper mechanically strong.

The frequency dependences of the standing wave ratio and reflection coefficient, which illustrate buckypaper-electromagnetic radiation interaction in various wavelength intervals, are shown in Figs. 6 and 7. The coefficients of radiation attenuation by the buckypaper are listed in the table. It was found that the screening efficiency at $10 \mathrm{GHz}$ was about $28 \mathrm{~dB}$ for buckypaper [30] and about $16 \mathrm{~dB}$ for a polyurethaneCNT composite [31].
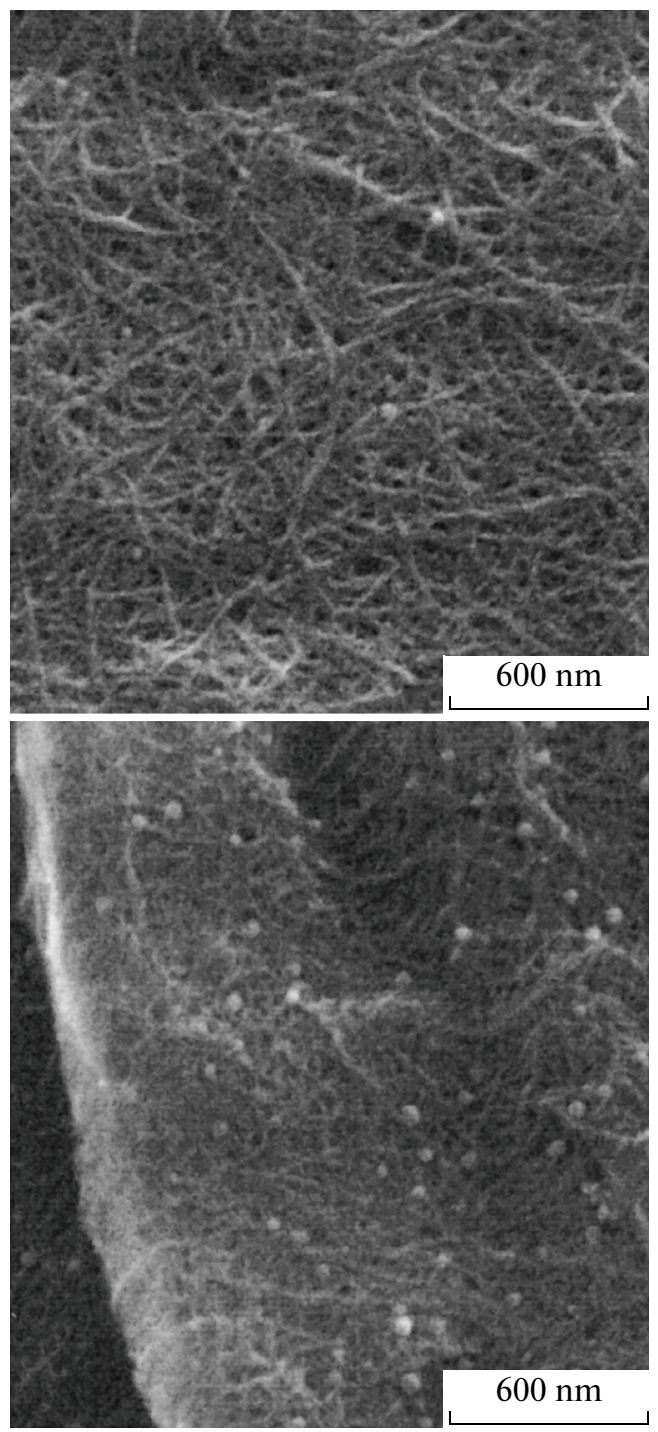

Fig. 5. SEM images of the buckypaper surface.

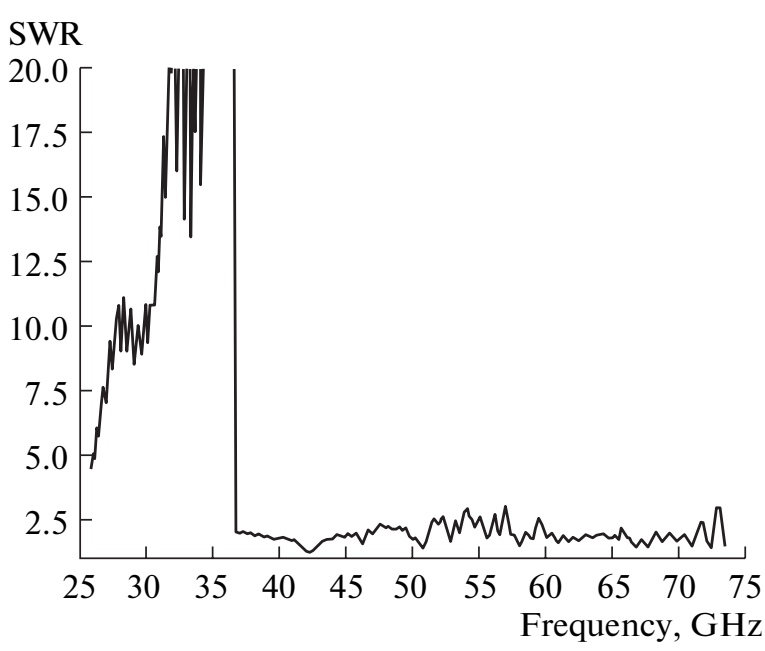

Fig. 6. Standing wave ratio (SWR) vs. frequency in the range $25.5-73.3 \mathrm{GHz}$ for the buckypaper.

TECHNICAL PHYSICS Vol. $56 \quad$ No. 11

2011 


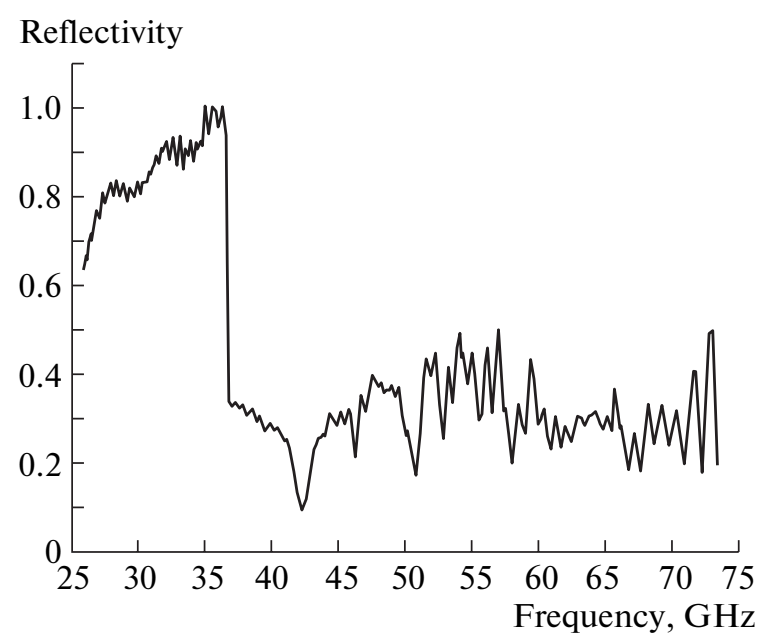

Fig. 7. Electromagnetic radiation reflection coefficient vs. frequency in the range $25.5-73.3 \mathrm{GHz}$ for the buckypaper.

The measured efficiency of screening of electromagnetic radiation with a frequency in the interval 0.05-13.50 GHz by a multiwall CNT-PMMA composite [26] increased with the weight fraction of multiwall CNTs increasing from 0.1 to $40 \mathrm{wt} \%$. The thickness of the composite varied between 60 and $165 \mu \mathrm{m}$. The highest efficiency of screening by a thick composite film was about $27 \mathrm{~dB}$. It was noted [26] that such a screening material is promising for commercial use.

With regard to the thickness of the buckypaper samples $(\approx 30 \mu \mathrm{m})$, the above data suggest strong interaction between buckypaper and electromagnetic radiation. This can be naturally explained by the high conductivity of CNTs. We can thus conclude that buckypaper is a well conducting material and, being a system of very thin entwined conductors (nanowires), effectively interacts with radiation. As has been mentioned above, tubes with metallic conductivity prevailed in the CNT array used to prepare buckypaper. For the electromagnetic radiation wavelengths considered in this paper, the buckypaper-radiation interaction is also similar to radiation attenuation by thin metallic layers, most of the radiation being reflected by the surface of the material. This is especially true for electromagnetic radiation with frequencies in the range $25.5-36.8 \mathrm{GHz}$.

Attenuation of electromagnetic radiation by buckypaper in different frequency ranges

\begin{tabular}{c|c}
\hline Frequency range, $\mathrm{GHz}$ & Mean attenuation, $\mathrm{dB}$ \\
\hline $25.5-36.8$ & 20 \\
$36.3-53.42$ & 37 \\
$53.41-74.3$ & 41 \\
\hline
\end{tabular}

\section{CONCLUSIONS}

An array of mostly metallic single-wall CNTs $0.8-$ $0.9 \mathrm{~nm}$ in diameter was synthesized by decomposing carbon monoxide with iron carbonyl used as a catalyst.

Structurally, the material obtained by depositing CNTs from a solution on a filter is very similar to normal black paper (it was given the name buckypaper). When depositing, CNT bundles intertwine, forming a dense stable structure. At room temperature, buckypaper strongly interacts with electromagnetic radiation with a frequency in the interval $25.5-73.3 \mathrm{GHz}$. The attenuation reaches $40 \mathrm{~dB}$, which renders this material promising for protection against electromagnetic radiation in sensitive devices and systems, especially in those experiencing elevated mechanical and thermal loads.

\section{REFERENCES}

1. P. N. D'yachkov, Carbon Nanotubes: Structure, Property, Application (BINOM, Moscow, 2006) [in Russian].

2. A. G. Tkachev and I. V. Zolotukhin, Equipment and Methods for Synthesis of Carbon Nanostructures: Monograph (Mashinostroenie-1, Moscow, 2007) [in Russian].

3. L. Berhan, Y. B. Yi, A. M. Sastry, E. Munoz, M. Selvidge, and R. Baughman, J. Appl. Phys. 95, 4335 (2004).

4. J. G. Park, J. Smithyman, Ch.-Y. Lin, A. Cooke, A. W. Kismarahardja, Sh. Li, R. Liang, J. S. Brooks, Ch. Zhang, and B. Wang, Appl. Phys. 106, 104310 (2009).

5. Z. Spitalsky, Ch. Aggelopoulos, G. Tsoukleri, Ch. Tsakiroglou, J. Parthenious, S. Georga, C. H. Krontiras, D. Tasis, K. Papagelis, and C. Galiotis, Mater. Sci. Eng. B 165, 135 (2009).

6. P. Connet, Z. Liang, E.-S. Choi, R. S. Kadambala, C. Zhang, J. S. Brooks, B. Wang, and L. Kramer, Curr. Appl. Phys. 6, 1 (2005).

7. D. Wang, Nanotechnology 19, 075608 (2008).

8. X. W. Tang, Y. Yang, W. Kim, Q. Wang, P. Qi, and H. Dai, Phys. Med. Biol. 50 (23), (2005).

9. B. W. Smith and D. E. Luzzi, J. Appl. Phys. 90, 3509 (2001) .

10. J. A. Astrom, A. V. Krasheninnikov, and K. Nordlund, Phys. Rev. Lett. 93, 215503 (2004).

11. A. V. Krasheninnikov and K. Nordlund, Nucl. Instrum. Methods Phys. Res. B 216, 355 (2004).

12. Z. Ni, Q. Li, L. Yan, J. Gong, and D. Zhu, Carbon 46, 365 (2008).

13. A. Ishag, L. Yan, and D. Zhu, Nucl. Instrum. Methods Phys. Res. B 267, 1779 (2009).

14. C. D. Cress, Ch. M. Schauerman, B. J. Landi, S. R. Messenger, R. P. Raffaelle, and R. J. Walters, J. Appl. Phys. 107, 014316 (2010).

15. J. G. Park,S. Li, X. Fan, C. Zhang, and B. Wang, Nanotechnology 19, 185710 (2008). 
16. L. Wang, W. Chen, D. Xu, B. S. Shim, Y. Zhu, F. Sun, L. Liu, Ch. Peng, Zh. Jin, Ch. Xu, and N. A. Kotov, Nano Lett. 9, 4147 (2009).

17. P. Dharap, Zh. Li, S. Nagarajaiah, and E. V. Barrero, Nanotechnology 15, 379 (2004).

18. R. H. Baughman, C. Cui, A. A. Zakhidov, Z. Igbal, J. N. Barisci, G. M. Spinks, G. G. Wallace, D. De Rossi, A. G. Rinzler, O. Jaschinski, S. Roth, and M. Kertesz, Science 284, 1340 (1999).

19. A. Ohlan, K. Singh, A. Chandra, V. N. Singh, and S. K. Dhawan, J. Appl. Phys. 106, 044305 (2009).

20. B. Hornbostel, U. Leute, P. Potdchke, J. Kotz, D. Kornfeld, P. Chiu, and S. Poth, Physica E 40, 2425 (2008).

21. L. V. Ruitao, F. Kang, J. Gu, X. Gui, J. Wei, K. Wang, and D. Wu, Appl. Phys. Lett. 93, 223105 (2008).

22. L. Zhang, H. Zhu, Y. Song, Y. Zhang, and Y. Huang, Mater. Sci. Eng., B 152, 78 (2008).

23. J. A. Roberts, T. Imholt, Z. Ye, C. B. Dyke, D. W. Price, and J. M. Tour, J. Appl. Phys. 95, 4352 (2004).

24. N. Li, Y. Huang, F. Du, Y. He, X. Lin, H. Gao, Y. Ma, F. Li, and Y. Chen, Nano Lett. 6, 1141 (2006).

25. P. Kuzhir, S. Maksimenko, D. Bychanok, V. Kuznetsov, S. Moseenkov, I. Mazov, O. Shenderova, and $\mathrm{Ph}$. Lambin, Metamaterials 3, 148 (2009).

26. H. M. Kim, K. Kim, C. Y. Lee, J. Joo, S. J. Cho, S. Yoon, O. A. Pejakovic, J. W. Yoo, and A. J. Epstein, Appl. Phys. Lett. 84, 589 (2004).

27. S. H. Park, P. Thielemann, P. Asbeck, and P. R. Bandaru, Appl. Phys. Lett. 94, 243111 (2009).
28. H. Zhang, G. Zeng, Y. Ge, T. Chen, and L. Hu, J. Appl. Phys. 105, 054314 (2009).

29. V. E. Muradyan, E. A. Sokolov, S. D. Babenko, and A. P. Moravskii, Zh. Tekh. Fiz. 80 (2), 83 (2010) [Tech. Phys. 55, 242 (2010)].

30. H. Xu, S. M. Anlage, L. Hu, and G. Gruner, Appl. Phys. Lett. 90, 183119 (2007).

31. Z. Liu, G. Bai, Y. Huang, Y. Ma, F. Du, F. Li, T. Guo, and Y. Chen, Carbon 45, 821 (2007).

32. F. F. Komarov, M. P. Samtsov, V. B. Karpovich, E. Yu. Leshchenko, I. K. Kirina, Vestn. Belarusk. Gos. Univ., Ser. 1, No. 3, 18 (2005).

33. F. F. Komarov, O. V. Mil'chanin, E. Munoz, V. N. Yuvchenko, and S. S. Grechnyi, Dokl. Akad. Nauk Belarusi 48 (6), 39 (2004).

34. A. G. Rinzler, J. Liu, H. Dai, P. Nikolaev, C. B. Huffman, F. J. Rodriguez-Macias, P. J. Boul, A. H. Lu, D. Heymann, D. T. Colbert, R. S. Lee, J. E. Fischer, A. M. Rao, P. C. Eklund, and R. E. Smalley, Appl. Phys. A 67, 29 (1998).

35. H. Kataura, Y. Kumazawa, Y. Maniwa, et al., Synth. Met. 103, 2555 (1999).

36. A. M. Rao, E. Richter, Sh. Bandow, et al., Science 275, 187 (1997).

37. Sh. Bandow, S. Asaka, Y. Saito, et al., Phys. Rev. Lett. 80, 3779 (1998).

38. I. W. Chiang, B. E. Brinson, A. Y. Huang, et al., J. Phys. Chem. B 105, 8297 (2001).

39. J. W. G. Wildoer, L. C. Venema, A. G. Rinzler, et al., Nature 391, 59 (1998).

\section{SPELL OK}

